\title{
A beacon-enabled least-time and energy efficient with one-level data aggregation routing protocol for WSNs using IEEE 802.15.4
}

\begin{abstract}
The Wireless Sensor Networks (WSNs) field of research is an interesting topic in the research community these days, because of its applicability in various fields such as civilian and medical research applications. Due to the resources and energy constraints in WSNs, routing can be considered as one of the most important issues in these networks. Every routing protocol designed for WSNs should be reliable, energy-efficient and prolong the network lifetime. This research proposes a beacon-enabled least-time and energy-efficient routing protocol with one-level data-aggregation using an IEEE 802.15.4 which is suitable for LowRate Wireless Personal Area Networks as WSNs, because of its low power consuming feature. The proposed protocol is compared to popular ad hoc and WSNs routing protocols i.e., Ad hoc On-Demand Distance Vector, Dynamic Source Routing, Destination-Sequenced Distance Vector routing, Directed Diffusion and Minimum Cost Forwarding. The propose work is simulated using network simulator 2. The simulation results show that the proposed protocol outperformed the routing protocols in the literature in terms of latency, throughput, average energy consumption and average network lifetime.
\end{abstract}

Keyword: Wireless sensor networks; Routing protocols; Energy efficient; IEEE 802.15.4; Ns2 